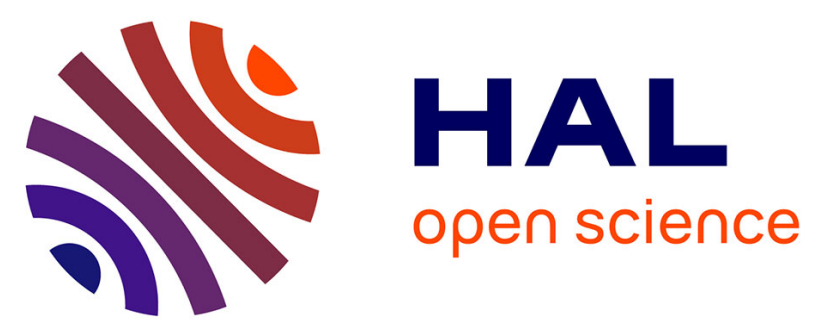

\title{
A sensor fusion approach for inertial sensors based 3D kinematics and pathological gait assessments: toward an adaptive control of stimulation in post-stroke subjects
}

Benoît Sijobert, François Feuvrier, Jérôme Froger, David Guiraud, Christine Azevedo Coste

\section{To cite this version:}

Benoît Sijobert, François Feuvrier, Jérôme Froger, David Guiraud, Christine Azevedo Coste. A sensor fusion approach for inertial sensors based 3D kinematics and pathological gait assessments: toward an adaptive control of stimulation in post-stroke subjects. EMBC: Engineering in Medicine and Biology Conference, Jul 2018, Honolulu, United States. pp.3497-3500, 10.1109/EMBC.2018.8512985 . hal01924841

\section{HAL Id: hal-01924841 \\ https://hal.inria.fr/hal-01924841}

Submitted on 16 Nov 2018

HAL is a multi-disciplinary open access archive for the deposit and dissemination of scientific research documents, whether they are published or not. The documents may come from teaching and research institutions in France or abroad, or from public or private research centers.
L'archive ouverte pluridisciplinaire HAL, est destinée au dépôt et à la diffusion de documents scientifiques de niveau recherche, publiés ou non, émanant des établissements d'enseignement et de recherche français ou étrangers, des laboratoires publics ou privés. 


\title{
A sensor fusion approach for inertial sensors based 3D kinematics and pathological gait assessments: toward an adaptive control of stimulation in post-stroke subjects*
}

\author{
B. Sijobert, F.Feuvrier, J.Froger, D.Guiraud, Member, IEEE EMBS and C.Azevedo Coste
}

\begin{abstract}
Pathological gait assessment and assistive control based on functional electrical stimulation (FES) in post-stroke individuals, brings out a common need to robustly quantify kinematics facing multiple constraints.

This study proposes a novel approach using inertial sensors to compute dorsiflexion angles and spatio-temporal parameters, in order to be later used as inputs for online close-loop control of FES. 26 post-stroke subjects were asked to walk on a pressure mat equipped with inertial measurement units (IMU) and passive reflective markers. A total of 930 strides were individually analyzed and results between IMU-based algorithms and reference systems compared. Mean absolute (MA) errors of dorsiflexion angles were found to be less than $4^{\circ}$, while stride lengths were robustly segmented and estimated with a MA error less than $10 \mathrm{~cm}$. These results open new doors to rehabilitation using adaptive FES closed-loop control strategies in "foot drop" syndrome correction.
\end{abstract}

\section{INTRODUCTION}

Being able to observe and assess a pathological motion is a recurrent need for practitioners. After a stroke, individuals are often hampered by walking difficulties [1]. Step-by-step kinematic and spatio-temporal parameters have been shown to be clinically relevant markers of impaired walking performances in post-stroke subjects [2]. Partly related to a deficit of voluntary control, individuals with hemiparesis are often unable to perform complete ankle dorsiflexion during swing phase, resulting in poor foot clearance, slow gait speed and compensatory strategies while walking [3]. Between $20 \%$ and $50 \%$ of individuals with post-stroke hemiplegia would be affected by this commonly called "foot drop" syndrome [4]. Among different rehabilitation approaches, one conventional treatment consists in using functional electrical stimulation (FES) applied to the peroneal nerve in order to elicit ankle dorsiflexion [5]. Classically, the stimulation is triggered by a heel switch inserted in the paretic side shoe (e.g. Odstock ${ }^{\odot}$ ODFS III) or by a tilt angle sensor (e.g. WalkAide ${ }^{\Theta}$, Bioness $\mathrm{L} 300^{\odot}$ ). Novel strategies to real-time modulate FES intensity or to regulate dorsiflexion in the presence of muscle state changes, such as fatigue and spasticity, could increase treatment efficiency and the number of potential users [6]. This brings out a common need of accurately quantifying kinematics in post-stroke

*This work was supported by a funding support (\#2015-2-41) from LabEx NUMEV (Montpellier, France) and Nîmes University Hospital (Nîmes, France).

B.Sijobert, D.Guiraud and C.Azevedo are with INRIA, Université de Montpellier, LIRMM, Montpellier, 34000 France (corresponding author to provide e-mail: benoit.sijobert@inria.fr).

F.Feuvrier and J.Froger are with Nîmes University Hospital (CHU), Nîmes, 30000 France (e-mail: jerome.froger@chu-nimes.fr). subjects, whether for assessing gait performance or for artificially controlling motion. Nevertheless, few systems are able to perform such ambulatory observations with a sufficient accuracy. Indeed, wearable sensors have been increasingly explored over the past few years as a mobile gait analysis solution. Numerous authors have suggested methods involving Inertial Measurement Units (IMU) sensors in order to estimate gait temporal or spatio-temporal parameters [7] as well as to compute joint angles during walking [8]. Inertial sensors basically measure acceleration, magnetic field and angular rate in their local coordinate system. Some devices directly provide the estimates of the sensor's orientation (in the form of quaternions, Euler angles or rotation matrices) with respect to a global fixed frame (e.g. Earth frame) by fusing sensors data. This is usually done by strap-down integrating the gyrometer values to obtain a first orientation, used then to remove Earth gravity from acceleration and combined with magnetometer measurements to provide azimuth [9]. Position can be afterward determined by double integrating acceleration without gravity. However, numerical integration introduces a drift error, while magnetic measurement can be disturbed by ferromagnetic materials, resulting in significant errors in the estimation of IMU attitude and position. During walking, a common solution is to update drift integration by simply zeroing the velocity during each detected stance phase, also called Zero Velocity Update (ZUPT) [10] or by using Attitude Heading and Reference System (AHRS) fusion methods proposed in the literature [11]. This requires to accurately segment gait cycle by detecting gait events (initial contact, toe off, etc...) from IMU data [12]. Only a few of the above mentioned methods have been compared to the gold standards (i.e. optical motion capture system or instrumented mats). In addition, they usually segment gait phases using angular velocity patterns, which is satisfying for normal gait [13] but tends to fail when gait is impaired and subjects develop compensatory strategies [14]. In this study, we propose a novel approach dedicated to pathological motion assessment from IMUs in 26 participants with post-stroke hemiplegia, integrating two aspects: 1) robust stance phase detection based on acceleration and angular rate combination and 2) estimation of joint angles based on an AHRS algorithm and gravity cancellation for reconstructing 3D trajectory of individual steps. The method has been validated by estimating 4 parameters initially defined as relevant by the practitioner to monitor rehabilitation progresses and to later adapt stimulation parameters: dorsiflexion angle at initial contact and at mid-swing instants, stride length and speed velocity. 


\section{MATERIAL AND METHODS}

\section{A. Experimental Protocol}

\section{1) Instrumentation}

Subjects were equipped with 4 IMUs (Fox HikoB ${ }^{\odot}$ Villeurbanne, France, L45mm x W36mm x H17mm, weight: $22 \mathrm{~g}$ ) featuring a 3 -axis accelerometer, a 3-axis magnetometer and a 3-axis gyrometer respectively mounted on the feet and shanks. Each IMU was strapped on a rigid support together with 4 reflective markers (Fig. 1a) tracked by an optical motion capture system (OMCS, Vicon ${ }^{\odot}$ Bonita MX) which cameras were installed along a Gaitrite ${ }^{\Theta}$ (CIR System Inc) walkway system (Fig. 1b).

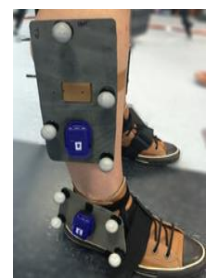

(a)

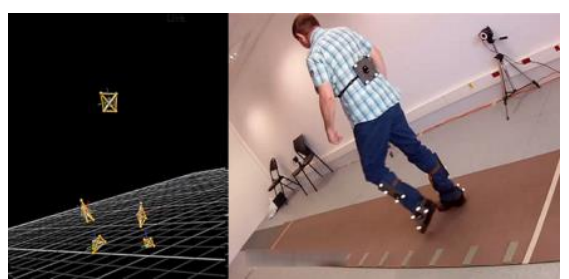

(b)
Figure 1. Each subject is equipped with IMUs strapped on rigid support together with 4 reflective markers (a) tracked by an optical motion capture system $\left(\operatorname{Vicon}^{\odot}\right)$. Subject is instructed to stand still for 5 seconds and then walk five meters on an instrumented walkway mat $\left(\right.$ Gaitrite $\left.^{\odot}\right)$, turn at the end of the carpet and walk back to initial position (b).

\section{2) Subjects}

This study included 29 subjects (mean $58.5 \pm 10.4$ years old; 9 females) after supratentorial ischaemic or hemorrhagic stroke, presenting a foot-drop, able to walk 10 meters without human help, with or without a walking stick. The protocol was approved by a national ethical committee (CPP) and by the local ethical committee of the University Hospital (CHU Nimes, France, RCB 2015-A00572-47), all subjects provided informed consent prior to the experiment.

\section{3) Stimulation}

In order to facilitate walking and elicit measurable dorsiflexion angles, subjects were also equipped with a classical "foot drop" stimulator (Odstock $^{\Theta}$ ODFS III) configured at the beginning of the experiment. Stimulation was triggered at heel off using a heel switch inside the shoe. Two electrodes of $23 \mathrm{~cm}^{2}$ delivered the stimulation either to the peroneal nerve or directly to the tibialis muscles of the affected side.

\section{4) Protocol conduct}

In order to best match Emory functional Ambulation Profile (E-FAP) test, participants were asked to walk five meters at a comfortable self-selected speed on the gait mat, to turn at the end of the carpet and walk back to their initial position. In case of technical issues or data loss, each trial was repeated three times to record at least one set of data by subject. IMUs, OMCS and gait carpet were synchronized at a hardware level via a trigger sent by the Gaitrite ${ }^{\Theta}$ to all the acquisition systems.

\section{B. Algorithms}

In the following formulas we use the quaternion notation $\mathrm{q}=\left(\mathrm{w}, \mathrm{x}_{\mathrm{i}}, \mathrm{y}_{\mathrm{j}}, \mathrm{z}_{\mathrm{k}}\right)$ defined in [15] as four scalar numbers, one real dimension $\mathrm{w}$ and an imaginary (or also called vector) part $\left(\mathrm{x}_{\mathrm{i}}, \mathrm{y}_{\mathrm{j}}, \mathrm{z}_{\mathrm{k}}\right)$.

The norm $\mathrm{N}$ of a quaternion is defined as: $\mathrm{N}(\mathrm{q})=$ $\overline{\mathrm{w}^{2}+\mathrm{x}^{2}+\mathrm{y}^{2}+\mathrm{z}^{2}}$. A quaternion with a unity norm is called unit quaternion. In the following algorithms, quaternions were systematically normalized as: $\mathrm{q}_{\text {unit }}=\frac{\mathrm{q}}{\operatorname{Norm}(\mathrm{q})}$ to be able to use unit quaternion properties. Let us note ${ }_{B}^{F} q$ the quaternion describing orientations of $\mathrm{B}$ relative to $\mathrm{F}$, for a united quaternion, ${ }_{B}^{F} \mathrm{q}$ equals ${ }_{F}^{B} \overline{\mathrm{q}}$, with $\overline{\mathrm{q}}$ defined as the quaternion conjugate $: \overline{\mathrm{q}}=\left(\mathrm{w},-\mathrm{x}_{\mathrm{i},},-\mathrm{y}_{\mathrm{j}},-\mathrm{z}_{\mathrm{k}}\right)$.

To compute quaternions from IMUs, AHRS fusion methods are usually based on nonlinear observers [16], [17], on a gradient descent method [18] or on estimators based on Extended Kalman Filters (EKF). In a previous work [19] we compared three different fusion algorithms to compute attitude from inertial measurement units and found out Martin et al. [17] was offering the best compromise between computational cost and reliability over time and magnetometer disturbances. Therefore, each quaternion representing the 3D orientation in global coordinate system was computed from $200 \mathrm{~Hz}$ sampled magneto-inertial data using Martin et al. nonlinear observer.

To reduce computational error, sensors were individually calibrated on each day of experimentation using Frosio et al. calibration model [20] for fitting in static conditions Earth magnetic field and gravity, incorporating not only bias and scale factor for each axis but also cross-axis symmetrical actors calculated through Gauss-Newton nonlinear optimization.

\section{1) Dorsiflexion angles}

Let us defined $\mathrm{q}_{\text {shank }}^{G}$ and $\mathrm{q}_{\text {foot }}^{G}$ the orientation of the shank and foot sensors in the global (i.e. ground) reference frame. The dorsiflexion angle corresponds to $\mathrm{q}_{\text {shank-foot }}$, the quaternion expressing rotations from shank to foot relative to the shank frame $S_{F}$ and computed using the following formula (Equ. 1), based on quaternion unit properties (Fig. 2):

$$
\mathrm{q}_{\text {shank-foot }}^{S_{F}}=\mathrm{q}_{\text {dors }}=\overline{\mathrm{q}}_{\text {shank }}^{G} * * \mathrm{q}_{\text {foot }}^{G}
$$

with $* *$ the specific quaternion multiplication, known as Hamilton product[15].

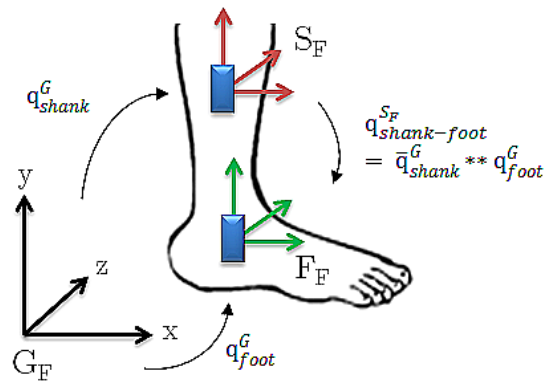

Figure 2. Dorsiflexion angle computation. The Hamilton product of the shank unit conjugate quaternion in global (Earth) frame by the quaternion of the foot also expressed in global frame gives the quaternion corresponding to the rotation of the foot relative to the shank. 
Table 1. Dorsiflexion angles RMS (root mean square) and MA (mean absolute) error at heel on and mid-swing, between IMU computation and Vicon. Spatio-temporal parameters compared between Gaitrite and IMUs computation.

\begin{tabular}{|c|c|c|c|c|}
\hline & RMSE & $\begin{array}{l}\text { MAE } \\
( \pm \text { SD })\end{array}$ & RMSE & $\begin{array}{l}\text { MAE } \\
( \pm \text { SD })\end{array}$ \\
\hline \multicolumn{3}{|c|}{ Dorsiflexion at heel on (initial contact) } & \multicolumn{2}{|c|}{$\begin{array}{c}\text { Stride length } \\
(\mathrm{cm})\end{array}$} \\
\hline All $(S=930)$ & $5.51^{\circ}$ & $\begin{array}{c}3.39^{\circ} \\
( \pm 3.37) \\
\end{array}$ & 12.64 & $\begin{array}{c}9.84 \\
( \pm 7.94) \\
\end{array}$ \\
\hline Paretic side ( $S=457$ ) & $4.89^{\circ}$ & $\begin{array}{c}3.12^{\circ} \\
( \pm 3.17)\end{array}$ & 12.61 & $\begin{array}{c}9.86 \\
( \pm 7.87)\end{array}$ \\
\hline Healthy side $(S=473)$ & $6.06^{\circ}$ & $\begin{array}{c}3.68^{\circ} \\
( \pm 3.56)\end{array}$ & 12.67 & $\begin{array}{c}9.81 \\
( \pm 8.02)\end{array}$ \\
\hline \multicolumn{3}{|c|}{ Dorsiflexion at mid-swing } & \multicolumn{2}{|c|}{ Speed $(\mathrm{cm} / \mathrm{s})$} \\
\hline All (S=930) & $5.01^{\circ}$ & $\begin{array}{c}3.74^{\circ} \\
( \pm 3.83)\end{array}$ & 6.17 & $\begin{array}{c}5.06 \\
( \pm 3.45)\end{array}$ \\
\hline Paretic side ( $\mathrm{S}=457$ ) & $4.89^{\circ}$ & $\begin{array}{c}3.73^{\circ} \\
( \pm 3.72)\end{array}$ & 6.22 & $\begin{array}{c}5.08 \\
( \pm 3.47)\end{array}$ \\
\hline Healthy side ( $S=473$ ) & $6.10^{\circ}$ & $\begin{array}{c}3.75^{\circ} \\
( \pm 3.95)\end{array}$ & 6.11 & $\begin{array}{c}5.06 \\
( \pm 3.43)\end{array}$ \\
\hline
\end{tabular}

At the beginning of each trial, subjects were asked to stand still during five seconds for getting rid of potential biases while defining zeros between IMU system and VICON system and for enabling Martin et al. algorithm to converge to an initial attitude.

\section{2) Trajectory}

Let us define ${ }_{B}^{G}$ p and ${ }_{B}^{G}$ v, the position and velocity of the feet sensors, respectively calculated by integrating twice and once the recorded acceleration without gravity $g$. Given $\mathrm{q}_{B}^{G}$, computed from [17] and unit quaternion properties $\mathrm{q}_{a c c}^{B}=$ $\left(0, a_{x}^{B}, a_{y}^{B}, a_{z}^{B}\right)$, we define the following formula (Equ. 2) to rotate acceleration vector and remove gravity $g$ :

$$
\mathrm{q}_{a c c}^{G}=\left(0, a_{x}^{G}, a_{y}^{G}, a_{z}^{G}\right)=\mathrm{q}_{B}^{G} * * \mathrm{q}_{a c c}^{B} * * \overline{\mathrm{q}}_{B}^{G}
$$

Therefore, to compute foot trajectory on each stride $n$ (Equ. 3):

where $\mathrm{a}_{G}=\left(\mathrm{a}_{x}^{G}, \mathrm{a}_{y}^{G}, \mathrm{a}_{z}^{G}\right)$

$$
{ }_{B}^{G} \mathrm{p}(\mathrm{t})=\iint_{\mathrm{t}_{\text {heel on }}(n-1)}^{\mathrm{t}_{\text {heel on }}(n)}\left(\mathrm{a}_{G}-g\right) d t
$$

The method described in [21] has been used to detect gait events, not only based on sagittal angular rate but also taking into account acceleration measurements.

Stride length was then defined as the distance between two consecutive initial contacts, computed in the transverse plane (Equ. 4):

$$
S L(n)=\sqrt{\begin{array}{l}
\left(p_{x}\left[\mathrm{t}_{h o}(n-1)\right]-p_{x}\left[\mathrm{t}_{h o}(n)\right]\right)^{2} \\
+\left(p_{z}\left[\mathrm{t}_{h o}(n-1)\right]-p_{z}\left[\mathrm{t}_{h o}(n)\right]\right)^{2}
\end{array}}
$$

\section{RESULTS}

29 subjects took part to the protocol. 3 subjects were finally too weak to perform any trial. Therefore the analysis and results refer to 26 subjects. A total of 930 strides (457 on paretic side and 473 on healthy side) were recorded. For each stride, the spatio-temporal parameters were computed from
IMUs and compared to parameters estimated from gold standard devices. Dorsiflexion angles were compared between IMUs and Vicon ${ }^{\odot}$ at mid-swing and heel-on, based on gait events instants extracted from Gaitrite ${ }^{\odot}$. Table 1 shows the results for all the analyzed strides: a Root Mean Square (RMS) error between OMCS and IMUs estimations of dorsiflexion of $5.51^{\circ}$ at initial contact and $5.01^{\circ}$ at midswing and a Mean Absolute (MA) error of respectively $3.39^{\circ}$ and $3.74^{\circ}$. The average dorsiflexion angle in all participants was $8.76^{\circ}$ at initial contact and $9.32^{\circ}$ at mid-swing. We observed a RMS error of $12.64 \mathrm{~cm}$ and a MA error of 9.84 $\mathrm{cm}$ regarding stride length estimations between Gaitrite ${ }^{\odot}$ and IMUs, and a RMS error of $6.17 \mathrm{~cm} / \mathrm{s}$ and MA error of 5,06 $\mathrm{cm} / \mathrm{s}$ for gait speed computation. The average stride length in all participants was $57.49 \mathrm{~cm}$ and the average gait speed $30.36 \mathrm{~cm} / \mathrm{s}$.

\section{DISCUSSION - CONCLUSION}

In this study, we compared gait variables estimated from IMUs data and gold standard systems. The initial aim was to analyze the reliability and accuracy of using IMUs to analyze motion, for later considering them as FES-based assistive control inputs. In our case, assessing pathological motion has been challenging and has required multiple non optimal choices from the data collection to the algorithm design. OMCS data acquisition suffered from many data losses and artefacts due to marker occultation by cane or markers breakage. As a result, we had to use rigid objects instead of a complete set of markers. Using a simple single strap, they were approximately positioned in sagittal plane, in order to improve visibility and ensure an eased installation time. An important advantage of our approach is the absence of specific procedure and preparation to locate sensors and use them. Except of a 5s static posture at the beginning of each trial, no calibration motions [8] were requested from the participants and no manual measurements of body dimensions had to be done nor individual adaptation. To estimate stride length and velocity for each step in each trial, feet trajectories have to be computed in a global frame. Usually, the solution consists in double integrating 2D linear acceleration in sagittal plane with an angular rate based gait cycle segmentation [13], [22] and an angular rate integration to estimate orientation needed to gravity removal. In our study, pathological gait was often associated to compensatory strategies (e.g. circumduction walk) and slow motions. These existing methods were not applicable to assess the gait of post-stroke subjects with a complex forward swing. Therefore, we had to adapt an algorithm we developed in an earlier work for Parkinson's disease [21], to segment impaired gait cycle and we took advantage of Martin et al. [17] quaternion computation to accurately remove gravity. Only a few studies have been conducted in hemiplegic participants in the literature [23], thus we mainly compared our results with publications on healthy individuals [24]. They seem in accordance with a MAE less than $4^{\circ}$ for the dorsiflexion angle and less than $10 \mathrm{~cm}$ for stride length estimation. 
One challenge of our study was to estimate very small dorsiflexion angles (about $10^{\circ}$ in all participants). To be able to compute dorsiflexion angle with an error of $4^{\circ}$ is a sufficient accuracy to detect the dorsiflexion tendency to decrease in the presence of muscle fatigue and to adapt FES parameters for counteracting fatigue effects.

Furthermore, we have designed the algorithms to be straight forward implemented for online use, as they intend to be used for FES control. To shorten calculation time and be able to track any orientations without singularities, we chose a quaternion representation computed from a low-cost observer-based attitude and heading reference system [17]. Euler angles well known gimbal lock may not be an issue when dealing with 3D rotations, but in our protocol it could have been a serious limitation when tracking lower limbs movements.

Finally, as we compared three different systems outputs (IMU, Gaitrite $^{\odot}$ and Vicon ${ }^{\odot}$ ) at different sampling rates but at a similar time mark (e.g. mid-swing), synchronization had to be accurately done not to introduce additional error.

These results break new grounds toward adaptive online control of the dorsiflexion. The complete knowledge of 3D trajectory and attitude of each stride could enable new closeloop strategies, such as adapting stimulation to obstacles (Fig. 3), stairs, or fatigue based on joint angle estimation.

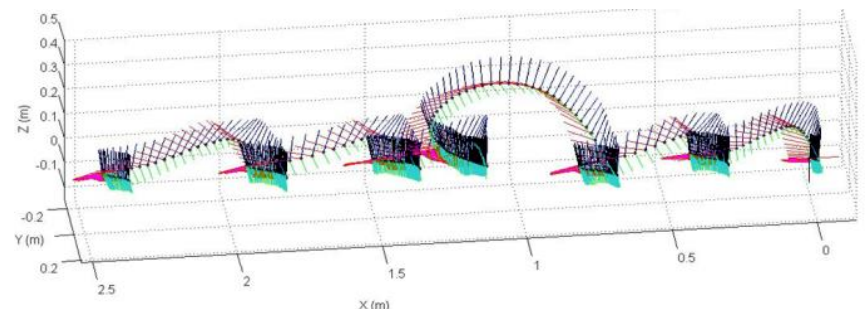

Figure 3. 3D trajectory of the foot IMU from a post-stroke subject obtained by integrating $3 \mathrm{D}$ accelerations with gravity removed from quaternion AHRS estimation. A Zero Velocity Update (ZUPT) is performed at each beginning and ending of the strides. In this example, the fourth stride corresponds to an obstacle crossing put on the gait mat. (x-IMU based Matlab 3D plotting : http://x-io.co.uk/gait-tracking-with-x-imu/)

\section{REFERENCES}

[1] S. M. Woolley, "Characteristics of Gait in Hemiplegia," Top Stroke Rehabil., vol. 7, no. 4, pp. 1-18, 2001.

[2] C. K. Balasubramanian, R. R. Neptune, and S. A. Kautz, "Variability in spatiotemporal step characteristics and its relationship to walking performance post-stroke," Gait Posture, vol. 29, no. 3, pp. 408-414, 2009.

[3] G. Chen, C. Patten, D. H. Kothari, and F. E. Zajac, "Gait differences between individuals with post-stroke hemiparesis and non-disabled controls at matched speeds," Gait Posture, vol. 22, no. 1, pp. 51-56, 2005.

[4] A. Kralj, R. Ačmović, and U. Stanič, "Enhancement of hemiplegic patient rehabilitation by means of functional electrical stimulation," Prosthet. Orthot. Int., vol. 17, no. 2, pp. 107-114, 1993.

[5] D. G. Everaert, R. B. Stein, G. M. Abrams, A. W. Dromerick, G. E. Francisco, B. J. Hafner, T. N. Huskey, M. C. Munin, K. J. Nolan, and C. V. Kufta, "Effect of a foot-drop stimulator and ankle-foot orthosis on walking performance after stroke: A multicenter randomized controlled trial," Neurorehabil. Neural Repair, vol. 27, no. 7, pp. 579-591, 2013.
C. Azevedo Coste, J. Jovic, R. Pissard-Gibollet, and J. Froger, "Continuous gait cycle index estimation for electrical stimulation assisted foot drop correction.," J. Neuroeng. Rehabil., vol. 11, no. 1, p. 118, Jan. 2014.

[7] R. Caldas, M. Mundt, W. Potthast, F. Buarque de Lima Neto, and B. Markert, "A systematic review of gait analysis methods based on inertial sensors and adaptive algorithms.," Gait Posture, vol. 57, pp. 204-210, Sep. 2017.

[8] T. Seel, J. Raisch, and T. Schauer, "IMU-Based Joint Angle Measurement for Gait Analysis," Sensors, vol. 14, no. 4, pp. 6891-6909, 2014.

[9] P. G. Savage, "Strapdown Inertial Navigation Integration Algorithm Design Part 1: Attitude Algorithms," J. Guid. Control. Dyn., vol. 21, no. 1, pp. 19-28, 1998.

[10] B. Mariani, C. Hoskovec, S. Rochat, C. Büla, J. Penders, and K. Aminian, "3D gait assessment in young and elderly subjects using foot-worn inertial sensors," J. Biomech., vol. 43, pp. 2999-3006, 2010.

[11] A. Cavallo, A. Cirillo, P. Cirillo, G. De Maria, P. Falco, C. Natale, and S. Pirozzi, "Experimental Comparison of Sensor Fusion Algorithms for Attitude Estimation," IFAC Proc. Vol., vol. 47, no. 3, pp. 7585-7591, Jan. 2014

[12] J. Rueterbories, E. G. Spaich, B. Larsen, and O. K. Andersen, "Methods for gait event detection and analysis in ambulatory systems," Med. Eng. Phys., vol. 32, no. 6, pp. 545-552, Jul. 2010. B. Sijobert, M. Benoussaad, J. Denys, R. Pissard-Gibollet, C. Geny, and C. Azevedo Coste, "Implementation and Validation of a Stride Length Estimation Algorithm , Using a Single Basic Inertial Sensor on Healthy Subjects and Patients Suffering from Parkinson 's Disease," Health (Irvine. Calif)., vol. 7, no. June, pp. 704-714, 2015.

[14] S. Yang, J. T. Zhang, A. C. Novak, B. Brouwer, and Q. Li, "Estimation of spatio-temporal parameters for post-stroke hemiparetic gait using inertial sensors," Gait Posture, vol. 37, no. 3, pp. 354-358, Mar. 2013.

[15] W. Hamilton, Lectures on Quaternions : containing a systematic statement of a new mathematical method. Dublin, 1853.

[16] R. Mahony, S. Member, T. Hamel, and J. Pflimlin, "Nonlinear Complementary Filters on the Special Orthogonal Group," vol. 53, no. 5, pp. 1203-1218, 2008.

[17] P. Martin and E. Salaün, "Design and implementation of a lowcost observer-based attitude and heading reference system," Control Eng. Pract., vol. 18, no. 7, pp. 712-722, Jul. 2010.

[18] S. O. H. Madgwick, A. J. L. Harrison, and R. Vaidyanathan, "Estimation of IMU and MARG orientation using a gradient descent algorithm," 2011 IEEE Int. Conf. Rehabil. Robot., pp. 17, 2011.

[19] B. Sijobert, R. Pissard-Gibollet, J. Froger, and C. A. Coste, "Validation of inertial sensor fusion algorithms in the context of hemiparetic gait assessment," in NER: Neural Engineering, 2015. I. Frosio, F. Pedersini, N. A. Borghese, and A. S. Model, "Autocalibration of MEMS Accelerometers," IEEE Trans. Instrum. Meas., vol. 58, no. 6, pp. 2034-2041, 2009.

[21] B. Sijobert, C. Azevedo, D. Andreu, C. Verna, and C. Geny, "Effects of Sensitive Electrical Stimulation-Based Somatosensory Cueing in Parkinson's Disease Gait and Freezing of Gait Assessment," Artif. Organs, vol. 41, no. 11, pp. E222-E232, Nov. 2017.

[22] M. Benoussaad, B. Sijobert, K. Mombaur, and C. Azevedo-Coste, "Robust Foot Clearance Estimation based on Acceleration Integration of Mounted Inertial Measurement Unit," Sensors, no. October 2015, pp. 1-13, 2015.

[23] D. Trojaniello, A. Cereatti, E. Pelosin, L. Avanzino, A. Mirelman, J. M. Hausdorff, and U. Della Croce, "Estimation of step-by-step spatio-temporal parameters of normal and impaired gait using shank-mounted magneto-inertial sensors : application to elderly, hemiparetic , parkinsonian and choreic gait," pp. 1-12, 2014. S. Yang and Q. Li, "Inertial Sensor-Based Methods in Walking Speed Estimation: A Systematic Review," Sensors, vol. 12, no. 5, pp. 6102-6116, Jan. 2012. 